Pacific

Journal of

Mathematics

\title{
THE RESIDUAL SPECTRUM OF INNER FORMS OF Sp(2)
}

TAKANORI YASUDA

Volume $232 \quad$ No. 2

October 2007 


\title{
THE RESIDUAL SPECTRUM OF INNER FORMS OF Sp(2)
}

\author{
TAKANORI YASUDA
}

\begin{abstract}
Let $G$ be the unitary group of the hyperbolic hermitian space with rank two over a quaternion division algebra over a totally real number field. We determine the irreducible decomposition of the residual discrete spectrum of $G$. Finally we give expected description of Arthur parameters associated to these representations.
\end{abstract}

\section{Introduction}

Let $k$ be a number field and $\boldsymbol{A}$ its adele ring. Let $G$ be a semisimple group defined over $k$. We write $L^{2}(G(k) \backslash G(A))$ for the Hilbert space of square-integrable functions on $G(k) \backslash G(A)$. The space $L_{0}^{2}(G(k) \backslash G(A))$ of cuspidal elements of $L^{2}(G(k) \backslash G(A))$ is contained in the discrete spectrum of $L^{2}(G(k) \backslash G(\boldsymbol{A}))$, and the orthogonal complement of $L_{0}^{2}(G(k) \backslash G(A))$ in the discrete spectrum is called the residual spectrum. In this paper we take as $G$ the unitary group of the twodimensional hyperbolic hermitian space over a quaternion division algebra $D$ over $k$. It is an inner form of $\operatorname{Sp}(2)$. We determine the irreducible decomposition of its residual spectrum, as a first example of a nonquasisplit group.

For $G=\operatorname{Sp}(2)$, the irreducible decomposition of its residual spectrum has been determined by Kim [1995]. Kon-no [1994] described it using theta correspondence in the case that $k$ is totally real. We have a decomposition:

$$
L^{2}(\operatorname{Sp}(2, k) \backslash \operatorname{Sp}(2, \boldsymbol{A}))=L^{2}(\operatorname{Sp}(2)) \oplus L^{2}\left(P_{0}\right) \oplus L^{2}\left(P_{1}\right) \oplus L^{2}\left(P_{2}\right),
$$

along constant terms [Mœglin and Waldspurger 1995, Prop. II.2.4]. Here $P_{0}$ is a Borel subgroup, $P_{1}$ and $P_{2}$ are Siegel and non-Siegel maximal parabolic subgroups, respectively. Then the residual spectrum can be described as

$$
L_{d}^{2}\left(P_{0}\right) \oplus L_{d}^{2}\left(P_{1}\right) \oplus L_{d}^{2}\left(P_{2}\right) .
$$

Here $L_{d}^{2}(\cdot)$ denotes the discrete spectrum of $L^{2}(\cdot)$. Similarly, the residual spectrum of our $G$ coincides with $L_{d}^{2}(P)$, where $P$ is a proper parabolic subgroup of $G$. $P$ corresponds to $P_{1}$ via the inner twist. Therefore we can make considerable use of the technique of decomposition of $L_{d}^{2}\left(P_{1}\right)$. Generally, the residual spectrum

MSC2000: primary 11F70, 11F72; secondary 11F27.

Keywords: residual spectrum, hermitian Morita theory, theta correspondence. 
is spanned by residues of the Eisenstein series associated with the cuspidal representations of the Levi factors of parabolic subgroups. A calculation of the poles of such Eisenstein series reduces to a calculation of normalization factors of certain intertwining operators. In the case of $\mathrm{Sp}(2)$ the Langlands-Shahidi normalization was used, where its normalization factors are written by automorphic $L$-functions. Therefore we can define the normalization factors for $P$ as an analogy of the case of $P_{1}$.

The residual spectrum for $G$ has a further decomposition,

$$
L_{d}^{2}(P)_{\infty} \oplus L_{d}^{2}(P)_{1},
$$

where $L_{d}^{2}(P)_{\infty}$ (resp. $\left.L_{d}^{2}(P)_{1}\right)$ is the space spanned by the residues of the Eisenstein series of infinite dimensional (resp. one-dimensional) cuspidal representations of a Levi factor $M$ of $P$. The irreducible decomposition of $L_{d}^{2}(P)_{\infty}$ is obtained by the Langlands classification (Theorem 4.1(2)); it is written in the form of the unique irreducible quotient of $\operatorname{Ind}_{P(\boldsymbol{A})}^{G(\boldsymbol{A})} \pi$ with an infinite dimensional cuspidal representation $\pi$ of $M$ satisfying some conditions. Then the representation replacing $\pi$ with the Jacquet-Langlands correspondence of $\pi$ appears in $L_{d}^{2}\left(P_{1}\right)$ [Kim 1995, Th. 3.3]. $L_{d}^{2}(P)_{1}$ contains the trivial representation (Theorem 4.1(1)). Every other irreducible constituent is described by the theta lift of the trivial representation of the unitary group of a (-1)-hermitian space over $D$ (Theorem 4.1(3)). This representation has a local component contained in $\operatorname{Ind}_{P\left(k_{v}\right)}^{G\left(k_{v}\right)}\left(\chi_{v}|\cdot|^{-1 / 2}\right) \circ v$ for any place $v$ of $k$, where $\chi_{v}$ is a quadratic character of $k_{v}^{\times}$and $v$ is the reduced norm of $D$. On the other hand, any irreducible constituent of $L_{d}^{2}\left(P_{0}\right)$ except for the trivial representation is the theta lift of the trivial representation of an orthogonal group; see [Kon-no 1994]. And this representation has a local component contained in

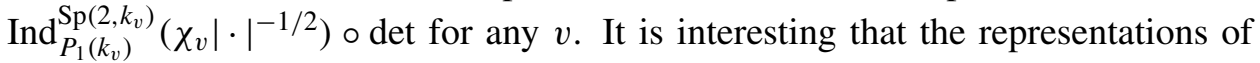
Theorem 4.1(3) do not have a multiplicity of one, unlike the case of $\operatorname{Sp}(2)$.

By Arthur's conjecture, an irreducible constituent of the residual spectrum should give the corresponding Arthur parameter. I expect that the Arthur parameters for the residual spectrum of $G$ are contained in those for the residual spectrum of $\mathrm{Sp}(2)$. It seems that the Arthur parameters for $L_{d}^{2}(P)_{\infty}$ appear for $L_{d}^{2}\left(P_{1}\right)$, and those for $L_{d}^{2}(P)_{1}$ appear for $L_{d}^{2}\left(P_{0}\right)$. I give the expected description of the Arthur parameters in Section 5.

\section{Preliminaries}

Let $k$ be a number field with adele ring $\boldsymbol{A}=\boldsymbol{A}_{k}$. We write $\boldsymbol{A}_{\infty}, \boldsymbol{A}_{f}$ for the infinite and finite components of $\boldsymbol{A}$, while ||$_{\boldsymbol{A}}$ denotes the idele norm of $\boldsymbol{A}^{\times}$. For any place $v$ of $k$ we write $k_{v}$ for the completion of $k$ at $v$, and ||$_{v}$ for the $v$-adic norm. If $v$ is finite, $O_{v}$ denotes the maximal compact subring of $k_{v}$. If $\psi$ is a nontrivial 
character of $\boldsymbol{A}$, trivial on $k$, and $v$ is a place of $k, \psi_{v}$ denotes the $v$-component of $\psi$.

Let $D$ be a quaternion division algebra over $k$. We write $v, \tau$, and $\iota$ for the reduced norm, the reduced trace, and the main involution of $D$, respectively. We write $D_{-}=\{x \in D \mid \tau(x)=0\}$. Also we write $S_{D}$ for the set of places $v$ of $k$ at which $D$ is ramified, and $s_{D}$ for the number of its elements, which is finite and even. We denote by $\mathbb{M}(n, R)$ the algebra of all $n \times n$-matrices over a ring $R$. Let $W=D^{\oplus 2}$ be the free left module over $D$ with rank two, and we equip it with the hermitian form $\langle$,$\rangle given by$

$$
\left\langle\left(x_{1}, y_{1}\right),\left(x_{2}, y_{2}\right)\right\rangle=x_{1}{ }^{l} y_{2}+y_{1}{ }^{\prime} x_{2} \quad\left(x_{1}, x_{2}, y_{1}, y_{2} \in D\right) .
$$

Writing $X:=\left\{(*, 0) \in D^{\oplus 2}\right\}, Y:=\left\{(0, *) \in D^{\oplus 2}\right\}$ we obtain a polarization of $W$. Let $G$ be the unitary group of this form, so that

$$
G(R)=\left\{g \in \mathrm{GL}\left(2, D \otimes_{k} R\right) \mid g\left(\begin{array}{ll}
0 & 1 \\
1 & 0
\end{array}\right)^{*} g=\left(\begin{array}{ll}
0 & 1 \\
1 & 0
\end{array}\right)\right\}
$$

for any commutative $k$-algebra $R$. Here we write $*\left(a_{i, j}\right)=\left({ }^{l} a_{j, i}\right)$ for $\left(a_{i, j}\right) \in$ $\mathbb{M}\left(2, D \otimes_{k} R\right) . G$ is nonquasisplit and is an inner form of $\operatorname{Sp}(2)$ with respect to a quadratic extension $k^{\prime}$ of $k$ such that all $v \in S_{D}$ do not split fully in $k^{\prime} / k$. Fix a $k$-parabolic subgroup $P$ and its Levi factor $M$ as

$$
P=\left\{\left(\begin{array}{r}
* * \\
*
\end{array}\right) \in G\right\}, \quad M=\left\{m(x):=\left(\begin{array}{cc}
x & 0 \\
0 & \left({ }^{(} x\right)^{-1}
\end{array}\right) \mid x \in D^{\times}\right\},
$$

where $D^{\times}$is regarded as an algebraic group over $k$ in the natural way. $P$ is the unique proper parabolic subgroup of $G$ up to $G(k)$-conjugate. We write again $v$ for the character of $M$ corresponding to the reduced norm via $m$. $U$ denotes the unipotent radical of $P$, so that

$$
U=\left\{u(y):=\left(\begin{array}{ll}
1 & y \\
0 & 1
\end{array}\right) \mid y \in D_{-}\right\} .
$$

Here $D_{-}$is also regarded as an algebraic group over $k$ in the natural way. The $k$-split component of the center of $M$ is

$$
A=\left\{a(t):=\left(\begin{array}{cc}
t & 0 \\
0 & t^{-1}
\end{array}\right) \mid t \in \mathbf{G}_{m}\right\} .
$$

The element $\alpha$ of the character group $X^{*}(A)$ of $A$ is defined by $\alpha(a(t))=t^{2}$. It is the unique positive root of $A$ with respect to $P$, and $\alpha^{\vee}=a$ is the attached coroot. The Weyl group $W_{G}$ of $A$ in $G$ is equal to $\left\{1, w_{0}\right\}$, where

$$
w_{0}=\left(\begin{array}{ll}
0 & 1 \\
1 & 0
\end{array}\right) .
$$


We fix a maximal compact subgroup $\boldsymbol{K}=\prod_{v} \boldsymbol{K}_{v}$ of $G(\boldsymbol{A})$ such that

$$
\boldsymbol{K}_{v}= \begin{cases}G\left(\mathbb{O}_{v}\right) & \text { if } v \notin S_{D} \text { and } v \text { is nonarchimedean, } \\ O(4) \cap G(\mathbb{R}) & \text { if } v \notin S_{D} \text { and } v \text { is real, } \\ U(4) \cap G(\mathbb{C}) & \text { if } v \text { is complex, } \\ O\left(v^{\oplus 2}\right) \cap G(\mathbb{R}) & \text { if } v \in S_{D} \text { and } v \text { is real, }\end{cases}
$$

and $\boldsymbol{K}_{v}$ is an $A$-good maximal compact subgroup if $v \in S_{D}$ and $v$ is nonarchimedean. Here $O\left(v^{\oplus 2}\right)$ is the orthogonal group of a quadratic form $W \otimes \mathbb{R} \ni$ $\left(x_{1}, x_{2}\right) \mapsto v\left(x_{1}\right)+v\left(x_{2}\right)$. Then we have the Iwasawa decomposition $G(\boldsymbol{A})=$ $P(\boldsymbol{A}) \boldsymbol{K}$. We write $\boldsymbol{K}_{\infty}$ for $\prod_{v \mid \infty} \boldsymbol{K}_{v} \subset G\left(\boldsymbol{A}_{\infty}\right)$.

\section{Decomposition of the $L^{2}$-inner product}

We will make use of the results of [Mœglin and Waldspurger 1995], henceforth abbreviated [MW]. Let $\left(M, \mathfrak{P}_{1}\right)$ and $\left(M, \mathfrak{P}_{2}\right)$ be two cuspidal pairs [MW, II.1.1]. For their Paley-Wiener sections $\phi_{1} \in P_{\left(M, \mathfrak{P}_{1}\right)}$ and $\phi_{2} \in P_{\left(M, \mathfrak{P}_{2}\right)}$ [MW, II.1.2], $\theta_{\phi_{1}}$ and $\theta_{\phi_{2}}$ denote the pseudo-Eisenstein series, respectively [MW, II.1.10]. They are elements of $L^{2}(G(k) \backslash G(\boldsymbol{A}))$ and if $\left(M, \mathfrak{P}_{1}\right)$ and $\left(M, \mathfrak{P}_{2}\right)$ are not $G(k)$-conjugate, they are orthogonal. If $\left(M, \mathfrak{P}_{1}\right)$ and $\left(M, \mathfrak{P}_{2}\right)$ are $G(k)$-conjugate then the $L^{2}$-inner product between them is described as follows [MW, Theorem II.2.1].

$$
\left\langle\theta_{\phi_{1}}, \theta_{\phi_{2}}\right\rangle=\int_{\substack{\pi \in \mathfrak{P}_{1} \\ \operatorname{Re}(\pi)=\lambda_{0}}} A\left(\phi_{1}, \phi_{2}\right)(\pi) d \pi,
$$

where

$$
A\left(\phi_{1}, \phi_{2}\right)(\pi)=\sum_{w \in W\left(\left(M, \mathfrak{P}_{1}\right),\left(M, \mathfrak{P}_{2}\right)\right)}\left\langle M(w, \pi) \phi_{1}(\pi), \phi_{2}(-w(\bar{\pi}))\right\rangle .
$$

Here all notation follows [MW]. $W\left(\left(M, \mathfrak{P}_{1}\right),\left(M, \mathfrak{P}_{2}\right)\right)$ is a subset of $W_{G}$ and $M(1, \pi)$ is the identity, and

$$
\left(M\left(w_{0}, \pi\right) \phi_{1}(\pi)\right)(g)=\int_{U(\boldsymbol{A})} \phi_{1}(\pi)\left(w_{0}^{-1} u g\right) d u \quad(g \in G(\boldsymbol{A})) .
$$

This integral converges absolutely at $\pi \in \mathfrak{P}_{1}$ such that $\left\langle\operatorname{Re}(\pi), \alpha^{\vee}\right\rangle \gg 0$ and $\lambda_{0}$ is chosen in this area. We have to extend $M\left(w_{0}, \pi\right)$ to $\left\langle\operatorname{Re}(\pi), \alpha^{\vee}\right\rangle \geq 0$ analytically and rewrite Equation (3-1).

For a cuspidal pair $(M, \mathfrak{P}), \pi \in \mathfrak{P}$, and $\phi \in P_{(M, \mathfrak{P})}, \phi(\pi)$ belongs to the space $A(U(\boldsymbol{A}) M(k) \backslash G(\boldsymbol{A}))_{\pi}$ (defined in [MW, II.1.1]). Here $\pi$ is decomposed into a restricted tensor product $\bigotimes_{v} \pi_{v}$ as a (Lie $\left.M\left(\boldsymbol{A}_{\infty}\right) \otimes_{\mathbb{R}} \mathbb{C}, M\left(\boldsymbol{A}_{\infty}\right) \cap \mathbf{K}_{\infty}\right) \times M\left(\boldsymbol{A}_{f}\right)$ module. If $\pi$ is infinite dimensional then it has a multiplicity of one in the space $A_{0}(M(k) \backslash M(\boldsymbol{A}))$ of cuspidal forms on $M(k) \backslash M(\boldsymbol{A})$ by Jacquet-Langlands theory. 
Also if $\pi$ is one-dimensional it has a multiplicity of one clearly. Thus we have

$$
A(U(\boldsymbol{A}) M(k) \backslash G(\boldsymbol{A}))_{\pi}=\operatorname{Ind}_{\boldsymbol{K} \cap M(\boldsymbol{A})}^{K} \pi=\bigotimes_{v} \operatorname{Ind}_{P\left(k_{v}\right)}^{G\left(k_{v}\right)} \pi_{v}
$$

as $\left(\right.$ Lie $\left.G\left(\boldsymbol{A}_{\infty}\right) \otimes_{\mathbb{R}} \mathbb{C}, \boldsymbol{K}_{\infty}\right) \times G\left(\boldsymbol{A}_{f}\right)$-modules. We have a decomposition:

$$
M\left(w_{0}, \pi\right)=\otimes_{v} M\left(w_{0}, \pi_{v}\right),
$$

where $M\left(w_{0}, \pi_{v}\right)$ is defined by

$$
\left[M\left(w_{0}, \pi_{v}\right) \phi_{v}\right](g)=\int_{U\left(k_{v}\right)} \phi_{v}\left(w_{0}^{-1} u g\right) d u \quad\left(g \in G\left(k_{v}\right), \phi_{v} \in \operatorname{Ind}_{P\left(k_{v}\right)}^{G\left(k_{v}\right)} \pi_{v}\right) .
$$

Their elementary properties are found in [Knapp and Stein 1980] for the archimedean case and [Silberger 1979] for the nonarchimedean case. This allows us to deduce analytic properties of $M\left(w_{0}, \pi\right)$ from those of the local components $M\left(w_{0}, \pi_{v}\right)$. We define the normalization factor for $M\left(w_{0}, \pi_{v}\right)$ by

$$
\begin{aligned}
r\left(w_{0}, \pi_{v}, \psi_{v}\right) & =\frac{L_{w_{0}}\left(0, \pi_{v}\right)}{L_{w_{0}}\left(1, \pi_{v}\right) \varepsilon_{w_{0}}\left(0, \pi_{v}, \psi_{v}\right)}, \\
L_{w_{0}}\left(s, \pi_{v}\right) & =L\left(s, \pi_{v}\right) L_{k_{v}}\left(2 s, \omega_{\pi_{v}}\right), \\
\varepsilon_{w_{0}}\left(s, \pi_{v}, \psi_{v}\right) & =\varepsilon\left(s, \pi_{v}, \psi_{v}\right) \varepsilon_{k_{v}}\left(2 s, \omega_{\pi_{v}}, \psi_{v}\right),
\end{aligned}
$$

where $L\left(s, \pi_{v}\right), \varepsilon\left(s, \pi_{v}, \psi_{v}\right)$ denote the $L$ and $\varepsilon$-factor defined by Godement and Jacquet [1972], and $L_{k_{v}}\left(s, \omega_{\pi_{v}}\right)$ and $\varepsilon_{k_{v}}\left(s, \omega_{\pi_{v}}, \psi_{v}\right)$ denote the Hecke $L$ and $\varepsilon$ factor. For any $v \notin S_{D}$, this normalization factor coincides with the LanglandsShahidi normalization factor which is considered in [Shahidi 1990]. Let $\operatorname{Re}\left(\pi_{v}\right)$ denote the local analogue of $\operatorname{Re}(\pi)$.

Lemma 3.1. For any $v$, the normalized intertwining operator

$$
N\left(w_{0}, \pi_{v}, \psi_{v}\right)=r\left(w_{0}, \pi_{v}, \psi_{v}\right)^{-1} M\left(w_{0}, \pi_{v}\right)
$$

is holomorphic for $\left\langle\operatorname{Re}\left(\pi_{v}\right), \alpha^{\vee}\right\rangle \geq 0$.

Proof. If $v \notin S_{D}$ and $\pi_{v}$ is infinite dimensional then the lemma has already been shown by $\operatorname{Kim}\left[1995\right.$, p. 133-134]. If $v \in S_{D}$, then $\pi_{v}$ is square-integrable modulo the center, and therefore $M\left(w_{0}, \pi_{v}\right)$ is holomorphic and nonzero for $\left\langle\operatorname{Re}\left(\pi_{v}\right), \alpha^{\vee}\right\rangle \geq$ 0 . Since $r\left(w_{0}, \pi_{v}, \psi_{v}\right)$ does not vanish for $\left\langle\operatorname{Re}\left(\pi_{v}\right), \alpha^{\vee}\right\rangle \geq 0$, the lemma follows. Assume that $v \notin S_{D}$ and $\pi_{v}=\chi_{v} \circ v$, where $\chi_{v}$ is a quasi-character of $k_{v}^{\times}$. We write $B$ for the Borel subgroup of GL(2) consisting of upper triangular matrices. Identifying $M\left(k_{v}\right)$ with $\operatorname{GL}\left(2, k_{v}\right), \pi_{v}$ is the unique irreducible subrepresentation of $\operatorname{Ind}_{B\left(k_{v}\right)}^{M\left(k_{v}\right)}\left(\left.\left.\chi_{v}|\cdot|\right|_{v} ^{-1 / 2} \otimes \chi_{v}|\cdot|\right|_{v} ^{1 / 2}\right)$ [Jacquet and Langlands 1970]. We fix a set of positive roots of the root data for $\mathrm{Sp}(2) . r_{1}$ and $r_{2}$ denote the reflection attached 
to the short and long simple roots, respectively. We may assume that $w_{0}=r_{2} r_{1} r_{2}$. By the functional equation we have

$$
M\left(w_{0}, \rho[s]\right)=M\left(r_{2}, r_{1} r_{2} \rho[s]\right) \circ M\left(r_{1}, r_{2} \rho[s]\right) \circ M\left(r_{2}, \rho[s]\right),
$$

where $\rho[s]:=\left.\chi_{v}|\cdot|\right|_{v} ^{-1 / 2+s} \otimes \chi_{v}|\cdot|{ }_{v}^{1 / 2+s}$. It is known that

$$
\begin{gathered}
\frac{L_{k_{v}}\left(s+1, \chi_{v}|\cdot|{ }_{v}^{-1 / 2}\right) \varepsilon_{k_{v}}\left(s, \chi_{v}|\cdot|{ }_{v}^{-1 / 2}, \psi_{v}\right)}{L_{k_{v}}\left(s, \chi_{v}|\cdot|_{v}^{-1 / 2}\right)} M\left(r_{2}, r_{1} r_{2} \rho[s]\right), \\
\frac{L_{k_{v}}\left(2 s+1, \chi_{v}^{2}\right) \varepsilon_{k_{v}}\left(2 s, \chi_{v}^{2}, \psi_{v}\right)}{L_{k_{v}}\left(2 s, \chi_{v}^{2}\right)} M\left(r_{1}, r_{2} \rho[s]\right),
\end{gathered}
$$

and

$$
\frac{L_{k_{v}}\left(s+1, \chi_{v}|\cdot|{ }_{v}^{1 / 2}\right) \varepsilon_{k_{v}}\left(s, \chi_{v}|\cdot|_{v}^{1 / 2}, \psi_{v}\right)}{L_{k_{v}}\left(s,\left.\chi_{v}|\cdot|\right|_{v} ^{1 / 2}\right)} M\left(r_{2}, \rho[s]\right),
$$

are holomorphic in the region $\operatorname{Re}(s) \geq 0$. Since $N\left(w_{0}, \rho[s], \psi_{v}\right)$ is the composition of these three maps we obtain the lemma.

Take a $\phi(\pi)=\bigotimes_{v} \phi(\pi)_{v} \in P_{(M, \mathfrak{P})}$. Let $S$ be a finite set of places of $k$ including all the archimedean places such that at every $v \notin S, D, \pi$, and $\psi$ are unramified and $\phi(\pi)_{v}$ is the $K_{v}$-fixed vector with $\phi(\pi)_{v}(1)=1$. For any $v \notin S$ we have the Gindikin-Karpelevich formula [Langlands 1971, p.45]:

$$
M\left(w, \pi_{v}\right) \phi(\pi)_{v}=r\left(w, \pi_{v}, \psi_{v}\right) \phi(\pi)_{v} .
$$

Therefore

$$
\begin{aligned}
M\left(w_{0}, \pi\right) \phi(\pi) & =\bigotimes_{v \in S} r\left(w_{0}, \pi_{v}, \psi_{v}\right) N\left(w_{0}, \pi_{v}, \psi_{v}\right) \phi(\pi)_{v} \otimes \bigotimes_{v \notin S} r\left(w_{0}, \pi_{v}, \psi_{v}\right) \phi(\pi)_{v} \\
& =r\left(w_{0}, \pi\right) N\left(w_{0}, \pi\right) \phi(\pi)
\end{aligned}
$$

where

$$
\begin{aligned}
r\left(w_{0}, \pi\right) & :=\prod_{v} r\left(w_{0}, \pi_{v}, \psi_{v}\right), \\
N\left(w_{0}, \pi\right) \phi(\pi) & :=\bigotimes_{v \in S} N\left(w_{0}, \pi_{v}, \psi_{v}\right) \phi(\pi)_{v} \otimes \bigotimes_{v \notin S} \phi(\pi)_{v} .
\end{aligned}
$$

From Lemma 3.1, $M\left(w_{0}, \pi\right)$ is continued for $\left\langle\operatorname{Re}(\pi), \alpha^{\vee}\right\rangle \geq 0$ and the poles of $M\left(w_{0}, \pi\right)$ coincide with those of $r\left(w_{0}, \pi\right)$ in this region.

Proposition 3.2. Suppose that $M\left(w_{0}, \pi\right)$ has a pole $\pi^{\prime}$ for $\left\langle\operatorname{Re}(\pi), \alpha^{\vee}\right\rangle \geq 0$.

(i) If $\mathfrak{P}$ consists of infinite dimensional representations, $\pi^{\prime}$ is equal to $\pi_{0}|v|_{A}^{1 / 2}$, where $\pi_{0}$ is an irreducible self-dual cuspidal representation of $M(A)$ whose standard $L$-function $L\left(s, \pi_{0}\right)$ does not vanish at $s=1 / 2$. It is simple. 
(ii) If $\mathfrak{P}$ consists of one-dimensional representations then $\pi^{\prime}$ is equal to $|\nu|_{A}^{3 / 2}$ or $\left(\omega_{k^{\prime} / k}|\cdot|_{A}^{1 / 2}\right) \circ v$ where $k^{\prime} / k$ is a quadratic extension such that all $v \in S_{D} d o$ not split fully in $k^{\prime} / k$. It is simple.

Proof. (i) From Shahidi's nonvanishing theorem [1981, Theorem 5.1] and the Jacquet-Langlands theory, the only pole of $r\left(w_{0}, \pi\right)$ for $\left\langle\operatorname{Re}(\pi), \alpha^{\vee}\right\rangle \geq 0$ is $\pi_{0}|\nu|_{A}^{1 / 2}$ where $\omega_{\pi_{0}}$ is trivial and $L\left(s, \pi_{0}\right)$ does not vanish at $s=1 / 2$. Note that $\omega_{\pi_{0}}=\mathbf{1}_{D^{\times}(\boldsymbol{A})}$ implies $\pi_{0}$ is self-dual.

(ii) Let $\pi=\chi \circ v . L\left(s, \pi_{v}\right)$ is described as follows [Jacquet and Langlands 1970].

$$
L\left(s, \pi_{v}\right)= \begin{cases}L_{k_{v}}\left(s+1 / 2, \chi_{v}\right) L_{k_{v}}\left(s-1 / 2, \chi_{v}\right) & \text { if } v \notin S_{D}, \\ L_{k_{v}}\left(s+1 / 2, \chi_{v}\right) & \text { if } v \in S_{D} \text { and } v \text { is finite, } \\ 2(2 \pi)^{-(s+1 / 2)} \Gamma(s+1 / 2) & \text { if } v \in S_{D} \text { and } v \text { is real. }\end{cases}
$$

Thus a factor of $r\left(w_{0}, \pi\right)$ related to the poles is

$$
\left(\prod_{v \in S_{D}} L_{k_{v}}\left(-1 / 2, \chi_{v}\right)\right)^{-1} \cdot L_{k}(1 / 2, \chi) \cdot L_{k}\left(0, \chi^{2}\right) \cdot L_{k}\left(1, \chi^{2}\right)^{-1} .
$$

Here $L_{k}(\cdot, \cdot)$ denotes the Hecke $L$-function. We can calculate its poles easily.

Write $\mathfrak{S}\left(\pi_{0}\right)=\pi_{0}|v|_{A}^{1 / 2}$ for $\pi_{0}$ satisfying the condition of Proposition 3.2(i). Similarly write $\mathfrak{S}_{1}=|v|_{A}^{3 / 2}$ and $\mathfrak{S}_{\Theta}\left(k^{\prime}\right)=\left(\omega_{k^{\prime} / k}|\cdot|_{A}^{1 / 2}\right) \circ v$ for $\omega_{k^{\prime} / k}$ satisfying the condition of Proposition 3.2(ii). From [Harish-Chandra 1968, Lemma 101], $M\left(w_{0}, \pi\right)$ is bounded on any region of the form

$$
\left\{\pi \in \mathfrak{P} \mid 0 \leq\left\langle\operatorname{Re}(\pi), \alpha^{\vee}\right\rangle \leq R\right\}, \quad 0<R \in \mathbb{R} .
$$

Thus we can apply the residue theorem to (3-1). From Proposition 3.2 we have the following.

Theorem 3.3. Let $\left(M, \mathfrak{P}_{1}\right)$ and $\left(M, \mathfrak{P}_{2}\right)$ be cuspidal pairs, and $\theta_{\phi_{1}}\left(\phi_{1} \in P_{\left(M, \mathfrak{P}_{1}\right)}\right)$ and $\theta_{\phi_{2}}\left(\phi_{2} \in P_{\left(M, \mathfrak{P}_{2}\right)}\right)$ pseudo-Eisenstein series.

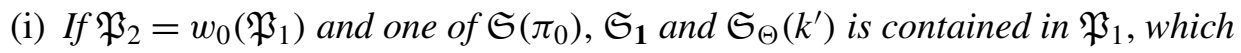
is denoted by $\mathfrak{S}$, then

$$
\left\langle\theta_{\phi_{1}}, \theta_{\phi_{2}}\right\rangle=\int_{\pi \in \mathfrak{P}_{1} \mid \operatorname{Re}(\pi)=0} A\left(\phi_{1}, \phi_{2}\right)(\pi) d \pi+c\left\langle N\left(w_{0}, \mathfrak{S}\right) \phi_{1}(\mathfrak{S}), \phi_{2}(\mathfrak{S})\right\rangle
$$

for some nonzero constant $c$.

(ii) Otherwise,

$$
\left\langle\theta_{\phi_{1}}, \theta_{\phi_{2}}\right\rangle=\int_{\pi \in \mathfrak{P}_{1} \mid \operatorname{Re}(\pi)=0} A\left(\phi_{1}, \phi_{2}\right)(\pi) d \pi
$$




\section{The residual spectrum of $G$}

We are now ready to determine the irreducible decomposition of the residual discrete spectrum of $G$.

For a $(-1)$-hermitian right $D$-space $(V, h), G(V)$ denotes the unitary group of $(V, h)$ and $G\left(V_{A}\right)$ the group of its adelic points. Set $\mathbb{W}=V \otimes_{D} W$ and $\langle\langle\rangle=$, $\frac{1}{2} \tau\left(h_{v} \otimes\langle\rangle,\right)$. Then $(\mathbb{W},\langle\langle\rangle$,$) is a symplectic space over k$. We will define the Weil representation $\omega_{\psi, V}$ of $G\left(V_{\boldsymbol{A}}\right) \times G(\boldsymbol{A})$ in Section 4.1.

Theorem 4.1. Let $k$ be a totally real number field. The irreducible constituents of the residual spectrum of $G$ consist of the following representations.

(i) The trivial representation $\mathbf{1}_{G(\boldsymbol{A})}$.

(ii) The unique irreducible quotient $J_{P}^{G}(\pi)$ of $\operatorname{Ind}_{P(\boldsymbol{A})}^{G(\boldsymbol{A})}\left(\pi|\nu|_{A}^{1 / 2}\right)$. Here $\pi$ runs over infinite dimensional irreducible self-dual cuspidal representations of $M(\boldsymbol{A})$ whose standard $L$-functions $L(s, \pi)$ do not vanish at $s=1 / 2$.

(iii) The theta lift $R(V)$ from the trivial representation of $G(V)$ under the Weil representation $\omega_{\psi, V}$. Here $V$ runs over local isometry classes of $(-1)$-hermitian right $D$-spaces with dimension one.

In the cases (1) and (2), the multiplicity of each representation is one. In the case (3), the multiplicity of each representation is $2^{s_{D}-2}$.

The proof of the theorem occupies the rest of this section. It is known that the discrete term, which is the second term on the right hand of (3-3), expresses the inner product for the residual spectrum [Mœglin and Waldspurger 1995, VI]. In other words, the residual spectrum of $G$ is isomorphic to the direct sum of the images of intertwining operators for all $\mathfrak{S}=\mathfrak{S}_{1}, \mathfrak{S}\left(\pi_{0}\right)$ and $\mathfrak{S}_{\Theta}\left(k^{\prime}\right)$ appearing in Proposition 3.2.

First assume that $\mathfrak{S}=\mathfrak{S}_{\mathbf{1}}$. We have that at each $v, \operatorname{Im} N\left(w_{0}, \mathfrak{S}_{v}\right)$ is isomorphic to $\mathbf{1}_{G\left(k_{v}\right)}$ by the Langlands classification. Thus the representation of Theorem 4.1(1) is obtained. Next assume that $\mathfrak{S}=\mathfrak{S}\left(\pi_{0}\right)$. For $v \notin S_{D}$, the proof of Proposition 3.2 in [Kim 1995] shows that $\operatorname{Ind}_{P\left(k_{v}\right)}^{G\left(k_{v}\right)} \mathfrak{S}_{v}$ has a unique irreducible quotient and $\operatorname{Im} N\left(w_{0}, \mathfrak{S}_{v}\right)$ is isomorphic to this quotient. If $v \in S_{D}, \mathfrak{S}_{v}$ is tempered so that $\operatorname{Im} N\left(w_{0}, \mathfrak{S}_{v}\right)$ is also isomorphic to the unique irreducible quotient of $\operatorname{Ind}_{P\left(k_{v}\right)}^{G\left(k_{v}\right)} \mathfrak{S}_{v}$. Therefore, all images of $N\left(w_{0}, \mathfrak{S}\right)$ are given by the representations of Theorem 4.1(2). We remark that the above results for $\mathfrak{S}=\mathfrak{S}_{1}$ and $\mathfrak{S}\left(\pi_{0}\right)$ do not need the fact that $k$ is totally real.

4.1. Construction by theta correspondence. Finally assume that $\mathfrak{S}=\mathfrak{S}_{\Theta}\left(k^{\prime}\right)$. At each place $v$ write $I\left(s, \chi_{v}\right)=\operatorname{Ind}_{P\left(k_{v}\right)}^{G\left(k_{v}\right)}\left(\left(\chi_{v}|\cdot|_{v}^{s}\right) \circ v\right)$, where $\chi_{v}$ is a character of $k_{v}^{\times}$ and $s \in \mathbb{C}$. Writing $\omega_{k_{v}^{\prime} / k_{v}}$ for the $v$-component of $\omega_{k^{\prime} / k}, \operatorname{Im} N\left(w_{0}, \mathfrak{S}_{\Theta}\left(k^{\prime}\right)_{v}, \psi_{v}\right)$ is a 
subrepresentation of $I\left(-1 / 2, \omega_{k_{v}^{\prime} / k_{v}}\right)$. We will describe the irreducible constituents of $I\left(-1 / 2, \omega_{k_{v}^{\prime} / k_{v}}\right)$ by using the theta correspondence.

(-1)-Hermitian spaces over quaternion algebras. We will review some basic facts about $(-1)$-hermitian spaces. The main involution $\iota$ of $D$ is of the first kind and of symplectic type [Scharlau 1985, p. 304]. For any place $v$ we again write $\iota$ for the involution of $D_{v}:=D \otimes_{k} K_{v}$ induced by the main involution of $D$, which also becomes of the first kind and of symplectic type. Therefore, from Remarks (iii) in the same location we can identify the local involution $\iota$ with the local main involution.

Let $F$ be a local field and $R$ be a quaternion algebra over $F$ with the main involution $\iota$. For $\Gamma=\left(\gamma_{i, j}\right) \in \mathbb{M}(m, R)$ such that $\left({ }^{l} \gamma_{j, i}\right)=-\Gamma\left(\operatorname{resp} .\left({ }^{l} \gamma_{j, i}\right)=\Gamma\right)$, a (-1)-hermitian (resp. hermitian) form on a right (resp. left) $R$-module $R^{\oplus m}$ (the set of column (resp. row) vectors) is defined by $\left(\left(v_{i}\right),\left(v_{j}^{\prime}\right)\right) \mapsto{ }^{t}\left({ }^{l} v_{i}\right) \Gamma\left(v_{j}^{\prime}\right)$ (resp. $\left.\left(\left(w_{i}\right),\left(w_{j}^{\prime}\right)\right) \mapsto\left(w_{i}\right) \Gamma^{t}\left({ }^{l} w_{j}^{\prime}\right)\right)$. We denote this form by $\langle\Gamma\rangle$. Similarly for $B \in \mathbb{M}(m, F)$ such that ${ }^{t} A=A$ (resp. ${ }^{t} A=-A$ ) we can define a quadratic (resp. symplectic) form $\langle B\rangle$ on $F^{\oplus m}$ (the set of column (resp. row) vectors).

- The case $R=\mathbb{M}(2, F)$. To observe $(-1)$-hermitian modules over $\mathbb{M}(2, F)$ we make use of an available theory which is called hermitian Morita theory. This implies an equivalence between the category of $(-1)$-hermitian (right) $R$-modules and the category of quadratic $F$-spaces. We will describe the correspondence in this theory ([Knus 1991] § I.9, [Scharlau 1985] p.361,362).

Let $(V, h)$ be a $(-1)$-hermitian right $R$-module. Set

$$
e=\left(\begin{array}{ll}
1 & 0 \\
0 & 0
\end{array}\right), e^{\prime}=\left(\begin{array}{ll}
0 & 1 \\
1 & 0
\end{array}\right) \in \mathbb{M}(2, F)=R,
$$

and $T_{V}=T_{(V, h)}:=V e$. A bilinear form $b_{V}=b_{(V, h)}$ on $T_{V}$ over $F$ is defined by

$$
h\left(v e, v^{\prime} e\right)=\left(\begin{array}{cc}
0 & 0 \\
b_{(V, h)}\left(v e, v^{\prime} e\right) & 0
\end{array}\right) \in \mathbb{M}(2, F) \quad \text { for } v, v^{\prime} \in V .
$$

Then $\left(T_{V}, b_{V}\right)$ is the quadratic space corresponding to $(V, h)$ in hermitian Morita theory. We define det $V$ as the determinant of $\left(T_{V}, b_{V}\right)$. For two $(-1)$-hermitian modules $\left(V_{1}, h_{1}\right),\left(V_{2}, h_{2}\right)$ and an isometry $\sigma:\left(V_{1}, h_{1}\right) \rightarrow\left(V_{2}, h_{2}\right)$, the restriction $\left.\sigma\right|_{T_{V_{1}}}: T_{V_{1}} \rightarrow T_{V_{2}}$ becomes an isometry from $\left(T_{V_{1}}, b_{V_{1}}\right)$ to $\left(T_{V_{2}}, b_{V_{2}}\right)$ as quadratic $F$-spaces. Therefore, the unitary group of $(V, h)$ is isomorphic to the orthogonal group of $\left(T_{V}, b_{V}\right)$.

In particular, consider the case of $(-1)$-hermitian free module with rank one. For a

$$
\Gamma=\left(\begin{array}{cc}
\alpha & \beta \\
\gamma & -\alpha
\end{array}\right) \in \mathbb{M}(2, F)_{-}
$$


we define $\mathcal{M}(\Gamma) \in \operatorname{Sym}_{2}(F)=\left\{\left.X \in \mathbb{M}(2, F)\right|^{t} X=X\right\}$ as

$$
\mu(\Gamma)=\left(\begin{array}{cc}
\gamma & -\alpha \\
-\alpha & -\beta
\end{array}\right)=J \Gamma .
$$

For a $(-1)$-hermitian right $R$-module, $(V, h)=(R,\langle\Gamma\rangle)$, one has that a quadratic space $\left(F^{\oplus 2},\langle\mathcal{M}(\Gamma)\rangle\right)$ is isometric to $\left(T_{V}, b_{V}\right)$. Note that $\operatorname{det} V=\operatorname{det} \mathcal{M}(\Gamma)=\operatorname{det} \Gamma$.

Hermitian Morita theory also implies the equivalence between the category of hermitian left $R$-modules and the category of symplectic $F$-spaces. Writing $\left(W_{F}, h_{F}\right)$ for the hermitian left $R$-module

$$
\left(R^{\oplus 2},\left\langle\left(\begin{array}{cc}
0 & 1_{2} \\
1_{2} & 0
\end{array}\right)\right\rangle\right),
$$

the Morita correspondence of $\left(W_{F}, h_{F}\right)$ is isometric to $\left(e W_{F}, s_{W_{F}}\right)$ where $s_{W_{F}}$ is defined by

$$
h_{F}\left(e w, e w^{\prime}\right)=\left(\begin{array}{cc}
0 & s_{W_{F}}\left(e w, e w^{\prime}\right) \\
0 & 0
\end{array}\right) \in \mathbb{M}(2, F) \quad \text { for } w, w^{\prime} \in W_{F} .
$$

Also, we have an isomorphism between the unitary group $G\left(W_{F}\right)$ of $\left(W_{F}, h_{F}\right)$ and $\operatorname{Sp}\left(e W_{F}\right)$ by restriction to $e W_{F}$.

- The case that $R$ is the quaternion division algebra over $F$. For a $(-1)$-hermitian right $R$-space $(V, h)$, the determinant det $V \in F^{\times} /\left(F^{\times}\right)^{2}$ is defined as the reduced norm of a matrix expression of $h$.

Proposition 4.2 [Scharlau 1985, Theorem 3.6, 3.7].

(a) Let $F$ be nonarchimedean.

(i) The isometry class of any regular (-1)-hermitian right $R$-space is determined by its dimension and determinant.

(ii) There exists a regular (-1)-hermitian right $R$-space with any dimension and determinant except for dimension 1 and determinant -1 .

(b) Let $F$ be real and archimedean.

(i) The isometry class of any regular (-1)-hermitian right $R$-space is determined by its dimension.

(ii) There exists a regular (-1)-hermitian right $R$-space with any dimension.

The next proposition is a statement about the local-global property with regard to $(-1)$-hermitian spaces. For a $(-1)$-hermitian right $D$-space $(V, h)$, the determinant det $V \in k^{\times} /\left(k^{\times}\right)^{2}$ is defined as the reduced norm of a matrix expression of $h$. For $\gamma \in D_{-} \backslash\{0\}$, let $k_{D, \gamma}^{\times}=\left\{c \in k^{\times} \mid\left(\gamma^{2}, c\right)_{v}=1\right.$ for all $\left.v \notin S_{D}\right\}$, where $(,)_{v}$ is the Hilbert symbol at $v$. A group homomorphism $\lambda$ is defined by

$$
k_{D, \gamma}^{\times} \ni c \mapsto\left\{\left(\gamma^{2}, c\right)_{v}\right\}_{v \in S_{D}} \in\{ \pm 1\}^{s_{D}} .
$$


Let $\{ \pm 1\}$ be regarded as the subgroup of $\{ \pm 1\}^{s_{D}}$ via the diagonal embedding. Note that the number of elements of $k_{D, \gamma}^{\times} / \lambda^{-1}(\{ \pm 1\})$ is $2^{s_{D}-2}$.

Proposition 4.3 [Scharlau 1985, Theorem 10.4.6, Remark 10.4.6]. Let (V, h) be a (-1)-hermitian right $D$-space.

(i) If $\operatorname{dim}_{D} V=1$ and $h=\langle\gamma\rangle$ for some $\gamma \in D_{-} \backslash\{0\}$, then for any $c \in k_{D, \gamma}^{\times},\langle c \gamma\rangle$ is locally isometric to $\langle\gamma\rangle$. For any $a \in \lambda^{-1}(\{ \pm 1\}),\langle a \gamma\rangle$ is globally isometric to $\langle\gamma\rangle$. Moreover

$$
\left\{\langle a \gamma\rangle \mid a \in k_{D, \gamma}^{\times} / \lambda^{-1}(\{ \pm 1\})\right\}
$$

is the set of classes locally isometric to $\langle\gamma\rangle$, so this set contains $2^{s_{D}-2}$ elements.

(ii) In general, for every dimension there exists exactly $2^{s_{D}-2}$ classes locally isometric to $(V, h)$.

Local theta correspondence. Let $(V, h)$ be a $(-1)$-hermitian right $D$-module with dimension $m$. We denote by $G(V)$ the unitary group of $V$ and, if $R$ is a $k$-algebra, by $G\left(V_{R}\right)$ the group of $R$-valued points of $G(V)$. For a place $v$, let $\left(V_{v}, h_{v}\right)$ be a $(-1)$-hermitian space over $D_{v}$. We define $G\left(V_{v}\right)$ similarly, but we often use $G\left(V_{v}\right)$ for the group of $k_{v}$-valued points of $G\left(V_{v}\right)$ by an abuse of notation. Let $\left(W_{v},\langle,\rangle_{v}\right)$ denote the completion of the hermitian space $(W,\langle\rangle$,$) over D$ at $v$. We define the Weil representation of $G\left(V_{v}\right) \times G\left(k_{v}\right)$ as follows.

Let $\mathbb{W}_{v}:=V_{v} \otimes_{D_{v}} W_{v}$ and $\left\langle\langle,\rangle_{v}:=\frac{1}{2} \tau\left(h_{v} \otimes \psi,\right\rangle_{v}\right)$. Then $\left(\mathbb{W}_{v},\langle\langle,\rangle\rangle_{v}\right)$ is a symplectic space over $k_{v}$ of dimension $8 m$.

- The case $v \notin S_{D}$. A homomorphism

$$
\mathbb{W}_{v}=V_{v} \otimes W_{v} \ni v \otimes w \mapsto v e^{\prime} e \otimes e e^{\prime} w+v e \otimes e w \in V_{v} e \otimes_{k_{v}} e W_{v}
$$

becomes an isometry between $\left(\mathbb{W}_{v},\langle\langle,\rangle\rangle_{v}\right)$ and $\left(V_{v} e \otimes_{k_{v}} e W_{v}, \frac{1}{2} b_{V_{v}} \otimes\left(-S_{W_{v}}\right)\right)$. On the other hand, a homomorphism

$$
O\left(V_{2} e, \frac{1}{2} b_{V_{v}}\right) \times \operatorname{Sp}\left(e W_{v},-S_{W_{v}}\right) \rightarrow \operatorname{Mp}\left(V_{v} e \otimes e W_{v}\right)
$$

is given in [Kudla 1994], where $\operatorname{Mp}\left(V_{v} e \otimes e W_{v}\right)$ denotes the metaplectic group. From this and Section 4.1 we have a homomorphism $G\left(V_{v}\right) \times G\left(k_{v}\right) \rightarrow \operatorname{Mp}\left(\mathbb{W}_{v}\right)$. Therefore the Weil representation $\omega_{\psi_{v}}$ of $\operatorname{Mp}\left(\mathbb{W}_{v}\right)$ induces a representation $\omega_{V_{v}}=$ $\omega_{\psi_{v}, V_{v}}$ of $G\left(V_{v}\right) \times G\left(k_{v}\right)$, which is realized on the space $\mathscr{S}\left(V_{v}\right)$ of Schwartz-Bruhat functions on $V_{v}=V_{v} \otimes X_{v}$.

- The case $v \in S_{D}$. A homomorphism $G\left(V_{v}\right) \times G\left(k_{v}\right) \rightarrow \operatorname{Mp}\left(\mathbb{W}_{v}\right)$ is given in [Kudla 1994]. The Weil representation $\omega_{\psi_{v}}$ of $\operatorname{Mp}\left(\mathbb{W}_{v}\right)$ induces a representation $\omega_{V_{v}}=\omega_{\psi_{v}, V_{v}}$ of $G\left(V_{v}\right) \times G\left(k_{v}\right)$, which is realized on $\mathscr{S}\left(V_{v}\right)=\mathscr{Y}\left(V_{v} \otimes X_{v}\right)$. 
For all places $v$ we have defined the Weil representation $\omega_{V_{v}}$ of $G\left(V_{v}\right) \times G\left(k_{v}\right)$. Some explicit formulae involving $\omega_{V_{v}}$ are as follows. Let $\phi \in \mathscr{S}\left(V_{v}\right)$ and $v \in V_{v}$.

$$
\begin{array}{ll}
\text { - } \omega_{V_{v}}\left(\left(\begin{array}{cc}
a & 0 \\
0 & \iota
\end{array} a^{-1}\right)\right) \phi(v)=\left((-1)^{m} \operatorname{det} V_{v}, v(a)\right)_{v}|a|_{k_{v}}^{m} \phi(v a) & \left(a \in D^{\times}\left(k_{v}\right)\right) \\
\text { - } \omega_{V_{v}}\left(\left(\begin{array}{ll}
1 & b \\
0 & 1
\end{array}\right)\right) \phi(v)=\psi_{v}\left(\frac{1}{4} \tau\left(b h_{v}(v)\right)\right) \phi(v) & \left(b \in D_{v,-}\right) \\
\text { - } \omega_{V_{v}}(h) \phi(v)=\phi\left(h^{-1} v\right) & \left(h \in G\left(V_{v}\right)\right)
\end{array}
$$

We will describe constituents of $I\left(-1 / 2, \chi_{v}\right)$. Let $\left(V_{v}, h_{v}\right)$ be a $(-1)$-hermitian (free) right module over $D_{v}$ with rank $m . \chi_{V_{v}}$ denotes the quadratic character of $k_{v}^{\times}$defined by $\chi_{V_{v}}(x)=\left((-1)^{m} \operatorname{det} V_{v}, x\right)_{v}$. For $v \notin S_{D}$ the description of the irreducible constituents of $I\left(-1 / 2, \chi_{v}\right)$ has been obtained by Kudla, Rallis, and Soudry [Kudla et al. 1992]. Therefore we will restrict to $v \in S_{D}$.

- The case of nonarchimedean $v \in S_{D}$. Consider the reducible points of $I\left(s, \chi_{v}\right)$, where $\chi_{v}$ is a quadratic character of $k_{v}^{\times}$.

Lemma 4.4. (i) If $\chi_{v}=1$ then $I\left( \pm 3 / 2, \chi_{v}\right)$ is reducible. $I\left(s, \chi_{v}\right)$ is irreducible for real $s \neq \pm 3 / 2$.

(ii) If $\chi_{v} \neq 1$ then $I\left( \pm 1 / 2, \chi_{v}\right)$ is reducible. $I\left(s, \chi_{v}\right)$ is irreducible for real $s \neq$ $\pm 1 / 2$.

Proof. The local Jacquet-Langlands correspondence of $\chi_{v} \circ v$ is $\sigma_{0}=\chi_{v} \circ \operatorname{det} \otimes \delta_{S t}$, where $\delta_{S t}$ denotes the Steinberg representation of GL $\left(2, k_{v}\right)$. By Proposition 2.1 in [Muić and Savin 2000] the Plancherel measure of $\chi_{v}$ coincides with the Plancherel measure of $\chi_{v} \circ \operatorname{det} \otimes \delta_{S t}$. Therefore, the poles and zeros of $\mu\left(s, \chi_{v} \circ v\right)$ coincide with the poles and zeros of

$$
\frac{L\left(1-s, \sigma_{0}^{\vee}\right) L_{k}\left(1-2 s, \omega_{\sigma_{0}}^{-1}\right) L\left(1+s, \sigma_{0}\right) L_{k}\left(1+2 s, \omega_{\sigma_{0}}\right)}{L\left(-s, \sigma_{0}^{\vee}\right) L_{k}\left(-2 s, \omega_{\sigma_{0}}^{-1}\right) L\left(s, \sigma_{0}\right) L_{k}\left(2 s, \omega_{\sigma_{0}}\right)},
$$

respectively. Since $\mu\left(0, \chi_{v} \circ v\right)=0$, the reducible points of $I\left(s, \chi_{v}\right)$ coincide with the poles of $\mu\left(s, \chi_{v} \circ v\right)$ [Silberger 1979]. All the poles are $s= \pm 3 / 2$ if $\chi_{v}=1$ and $s= \pm 1 / 2$ if $\chi_{v} \neq 1$.

In Proposition 3.2(ii), the local component $\omega_{k_{v}^{\prime} / k_{v}}$ at $v$ of $\omega_{k^{\prime} / k}$ is not trivial. Therefore we want the description of constituents of $I\left(-1 / 2, \chi_{v}\right)$ with a nontrivial $c h i_{v}$, using theta correspondence. We write $\mathscr{S}\left(V_{v}\right)_{G\left(V_{v}\right)}$ for the $G\left(V_{v}\right)$-coinvariant space of $S\left(V_{v}\right)$. Let $R\left(V_{v}\right)$ denote the image of the map

$$
\mathscr{Y}\left(V_{v}\right) \ni \phi \mapsto\left[G\left(k_{v}\right) \ni g \mapsto \omega_{V_{v}}(g) \phi(0)\right] \in I\left(m-3 / 2, \chi_{V_{v}}\right) .
$$

This map induces an isomorphism $\mathscr{S}\left(V_{v}\right)_{G\left(V_{v}\right)} \simeq R\left(V_{v}\right)$; see [Mœglin et al. 1987, Chap. 3 IV Th.7]. Let $\left(V_{v}^{\prime}, h_{v}^{\prime}\right)$ be a 1-dimensional $(-1)$-hermitian space with 
det $V_{v}^{\prime} \neq-1$. $\left(V_{v}^{\prime \prime}, h_{v}^{\prime \prime}\right)$ denotes the 2-dimensional (-1)-hermitian space given by $\operatorname{det} V_{v}^{\prime \prime}=-\operatorname{det} V_{v}^{\prime}$. Proposition 4.2 guarantees the existence and uniqueness of $\left(V_{v}^{\prime}, h_{v}^{\prime}\right)$ and $\left(V_{v}^{\prime \prime}, h_{v}^{\prime \prime}\right)$.

Proposition 4.5. (i) $R\left(V_{v}^{\prime}\right)$ and $R\left(V_{v}^{\prime \prime}\right)$ are the unique irreducible subrepresentations of $I\left(-1 / 2, \chi_{V_{v}^{\prime}}\right)$ and $I\left(1 / 2, \chi_{V_{v}^{\prime}}\right)$, respectively.

(ii) $N\left(w_{0},\left(\chi_{V_{v}^{\prime}}|\cdot|_{v}^{1 / 2}\right) \circ v, \psi_{v}\right)$ induces an isomorphism $I\left(1 / 2, \chi_{V_{v}^{\prime}}\right) / R\left(V_{v}^{\prime \prime}\right) \simeq$ $R\left(V_{v}^{\prime}\right)$.

Proof. The Jacquet module $I\left(-1 / 2, \chi_{V_{v}^{\prime}}\right)_{P}$ of $I\left(-1 / 2, \chi_{V_{v}^{\prime}}\right)$ along $P$ is

$$
\left(\chi_{V_{v}^{\prime}}|\cdot|_{v}^{1 / 2}\right) \circ v+\left(\chi_{V_{v}^{\prime}}|\cdot|_{v}^{-1 / 2}\right) \circ v
$$

in the Grothendieck group. On the other hand, $R\left(V_{v}^{\prime}\right)_{P} \simeq\left(\left.\chi_{V_{v}^{\prime}}|\cdot|\right|_{v} ^{-1 / 2}\right) \circ v$. Since $I\left(-1 / 2, \chi_{V_{v}^{\prime}}\right)$ has at most one proper $G\left(k_{v}\right)$-invariant space, the former of (i) follows. Similarly the latter of (i) is proved. From the Langlands classification we obtain (ii).

- The case of real archimedean $v \in S_{D}$. We have $G\left(k_{v}\right) \simeq \operatorname{Sp}(1,1)$. The reducible points of $I(s, \mathbf{1})$ have been obtained, as follows.

Lemma 4.6 [Johnson 1990, Corollary of Lemma 5.4]. If $s= \pm(2 n+1) / 2(n \in \mathbb{N})$ then $I(s, \mathbf{1})$ is reducible, otherwise $I(s, \mathbf{1})$ is irreducible. In particular $I( \pm 1 / 2, \mathbf{1})$ are irreducible.

Since $v\left(D_{v}^{\times}\right)=\mathbb{R}_{+}^{\times}$, we need not take the signed character in this lemma. Let $\mathscr{S}^{0}\left(V_{v}\right)$ denote the subspace of $\mathscr{Y}\left(V_{v}\right)$ of functions of the form $e^{-\pi v(x)} P(x)$ where $P$ is a polynomial on $V_{v}\left(\cong k_{v}^{\oplus 4 m}\right)$. This space is a $\left(\mathfrak{g}_{v}, \boldsymbol{K}_{v}\right)$-module where $\mathfrak{g}_{v}=$ Lie $G\left(k_{v}\right) \otimes_{\mathbb{R}} \mathbb{C}$. Fix an isomorphism $V_{v} \cong D_{v}^{\oplus m}$. We set $\mathfrak{h}_{v}=$ Lie $O^{*}(2 m) \otimes_{\mathbb{R}} \mathbb{C}$ and a maximal compact subgroup $L_{v}=O\left(v^{\oplus m}\right) \cap O^{*}(2 m)$ of $O^{*}(2 m)$ where $O\left(v^{\oplus m}\right)$ is the orthogonal group of $v^{\oplus m}$ ( $m$ times direct sum of the norm form on $D_{v}$ ). We write $\mathscr{S}^{0}\left(V_{v}\right)_{\left(\mathfrak{h}_{v}, L_{v}\right)}$ for the $\left(\mathfrak{h}_{v}, L_{v}\right)$-coinvariant space of $\mathscr{S}^{0}\left(V_{v}\right) . R\left(V_{v}\right)$ denotes the image of the map

$$
\mathscr{S}^{0}\left(V_{v}\right) \ni \phi \mapsto\left[G\left(k_{v}\right) \ni g \mapsto \omega_{V_{v}}(g) \phi(0)\right] \in I\left(m-3 / 2, \chi_{V_{v}}\right) .
$$

This map induces an isomorphism $\mathscr{S}^{0}\left(V_{v}\right)_{\left(\mathfrak{h}_{v}, L_{v}\right)} \simeq R\left(V_{v}\right)$ as $\left(\mathfrak{g}_{v}, \boldsymbol{K}_{v}\right)$-modules [Zhu 1992, Theorem II]. Let $\left(V_{v}^{\prime}, h_{v}^{\prime}\right)$ be a $(-1)$-hermitian space with dimension one. By Proposition 4.2 it is determined uniquely. From the above lemma and the Langlands classification we have the following.

Proposition 4.7. $N\left(w_{0},\left.|\cdot|\right|_{v} ^{1 / 2}, \psi_{v}\right)$ induces an isomorphism $I(1 / 2, \mathbf{1}) \simeq R\left(V_{v}^{\prime}\right)(=$ $I(-1 / 2, \mathbf{1}))$. 
We go back to a general place $v$. Let $\left(V_{v}, h_{v}\right)$ be a $(-1)$-hermitian right $D_{v^{-}}$ module with rank $m$. For $\beta \in D_{v,-}$, let $\Omega_{\beta}=\left\{v \in V_{v} \mid h_{v}(v, v)=\beta\right\}$. Also we write $\psi_{v, \beta}$ for the character of $U\left(k_{v}\right)$ given by $\psi_{v, \beta}(u(b))=\psi_{v}\left(\frac{1}{4} \tau(b \beta)\right)$.

Suppose $k_{v}$ is nonarchimedean. Set

$$
\mathscr{S}\left(V_{v}\right)_{\beta}=\mathscr{Y}\left(V_{v}\right) / \operatorname{Span}\left\{\omega_{V_{v}}(u) \varphi-\psi_{v, \beta}(u) \varphi \mid \varphi \in \mathscr{Y}\left(V_{v}\right), u \in U\left(k_{v}\right)\right\} .
$$

The next two lemmas are shown by an argument similar to the proof of [Rallis 1987, Lemma 4.2].

Lemma 4.8. If $\Omega_{\beta}=\phi$ then $R\left(V_{v}\right)_{\beta}=0$. If $v(\beta) \neq 0$ and $\Omega_{\beta} \neq \phi$ then $\operatorname{dim} R\left(V_{v}\right)_{\beta}=1$.

Suppose that $k_{v}$ is real. $I^{\infty}\left(s, \chi_{V_{v}}\right)$ denotes the smooth induced representation including $I\left(s, \chi_{V_{v}}\right)$ with its Fréchet topology. Let the topology of $\mathscr{S}\left(V_{v}\right)$ be given by the usual one. The map $i: \mathscr{S}^{0}\left(V_{v}\right) \rightarrow R\left(V_{v}\right) \subset I\left((m-3) / 2, \chi_{V_{v}}\right)$ extends to a continuous map

$$
i: \mathscr{S}\left(V_{v}\right) \rightarrow R^{\infty}\left(V_{v}\right) \subset I^{\infty}\left((m-3) / 2, \chi_{V_{v}}\right),
$$

where $R^{\infty}\left(V_{v}\right)$ is the image of $\mathscr{Y}\left(V_{v}\right)$. For $\beta \in D_{v,-}$, let $R^{\infty}\left(V_{v}\right)_{\beta}^{\prime}$ be the space of all continuous linear functionals $\mu$ on $R^{\infty}\left(V_{v}\right)$ such that $\mu(r(X) \Phi)=d \psi_{v, \beta}(X) \mu(\Phi)$ for all $X \in \mathfrak{u}=\operatorname{Lie} U(\mathbb{R})$ and all $\Phi \in R^{\infty}\left(V_{v}\right)$. Here $d \psi_{v, \beta}: \mathfrak{u} \rightarrow \mathbb{C}$ is the differential of $\psi_{v, \beta}$ and $r$ denotes the action on $R^{\infty}\left(V_{v}\right)$.

Lemma 4.9. If $\Omega_{\beta}=\phi$ then $R^{\infty}\left(V_{v}\right)_{\beta}^{\prime}=0$. If $v(\beta) \neq 0$ and $\Omega_{\beta} \neq \phi$ then $\operatorname{dim} R^{\infty}\left(V_{v}\right)_{\beta}^{\prime}=1$.

Global theta correspondence. For a $(-1)$-hermitian right $D$-module $(V, h)$ we write $\omega_{V}=\omega_{\psi, V}$ for the Weil representation of $G\left(V_{A}\right) \times G(\boldsymbol{A})$ on the space $\mathscr{Y}\left(V_{A}\right)$ of Schwartz-Bruhat functions on $V_{A}$. For any $\beta \in D_{-}$, we define a character $\psi_{\beta}$ of $U(\boldsymbol{A})$ by

$$
\psi_{\beta}(u(b))=\prod_{v} \psi_{v, \beta}\left(u\left(b_{v}\right)\right) \quad\left(u(b)=\left(u\left(b_{v}\right)\right) \in U(\boldsymbol{A})\right) .
$$

Let $\left\{\left(V_{v}, h_{v}\right)\right\}$ be a collection of $(-1)$-hermitian right $D_{v}$-modules with rank one for all $v$, and let $\Pi=\bigotimes_{v} R\left(V_{v}\right)$ (if it can be defined). $\Pi$ is regarded as a representation of (Lie $\left.G\left(\boldsymbol{A}_{\infty}\right) \otimes_{\mathbb{R}} \mathbb{C}, \boldsymbol{K}_{\infty}\right) \times G\left(\boldsymbol{A}_{f}\right)$, whose action is written by $r$. We write $\mathcal{W}_{\beta}(\Pi)$ for the space of linear functionals $\mu$ on $\Pi$ which satisfy

$$
\begin{aligned}
& \mu(r(u) f)=\psi_{\beta}(u) \mu(f) \quad\left(f \in \Pi, u \in U\left(\boldsymbol{A}_{f}\right)\right), \\
& \mu(r(X) f)=d \psi_{\beta}(X) \mu(f) \quad\left(X \in \operatorname{Lie} U\left(\boldsymbol{A}_{\infty}\right)\right),
\end{aligned}
$$

where $d \psi_{\beta}$ is the differential of the restriction of the character $\psi_{\beta}$ to $U\left(\boldsymbol{A}_{\infty}\right)$. For each archimedean place $v, R^{\infty}\left(V_{v}\right)$ denotes the closure of $R\left(V_{v}\right)$ in $I^{\infty}\left(1 / 2, \chi_{V_{v}}\right)$ 
as in the previous subsection. Let

$$
\Pi^{\infty}=\left(\bigotimes_{v \text { :arch }} R^{\infty}\left(V_{v}\right)\right) \otimes\left(\bigotimes_{v: \text { nonarch }} R\left(V_{v}\right)\right),
$$

which is a representation of $G(\boldsymbol{A})$. Let $\mathcal{W}_{\beta}^{\infty}\left(\Pi^{\infty}\right)$ be the space of all linear functionals $\mu$ on $\Pi^{\infty}$ which satisfy (4-1) and whose restrictions to $R^{\infty}\left(V_{v}\right)$ lie in $R^{\infty}\left(V_{v}\right)_{\beta}^{\prime}$. Let $\mathcal{W}_{\beta}^{\infty}(\Pi)$ be the subspace of $\mathcal{W}_{\beta}(\Pi)$ spanned by the restrictions of functionals in $\mathcal{W}_{\beta}^{\infty}\left(\Pi^{\infty}\right)$. We have the following proposition which is the same as Proposition 2.1 in [Kudla et al. 1992].

Proposition 4.10. Suppose that $\left\{\left(V_{v}, h_{v}\right)\right\}$ is given by the completions at all $v$ of some $(-1)$-hermitian right $D$-module $(V, h)$ with rank one and $\operatorname{det} V \neq 0$. We write $\Pi(V)=\bigotimes_{v} R\left(V_{v}\right)$. Let $O_{V}=\left\{\beta \in D_{-} \backslash\{0\} \mid h(x, x)=c \beta\right.$ for some $x \in V$ and some $c \in k_{D, \beta}^{\times}$.

(i) If $\beta \notin \mathcal{O}_{V}$ and $\beta \neq 0$ then $\mathscr{W}_{\beta}^{\infty}(\Pi(V))=0$.

(ii) If $\beta \in \mathcal{O}_{V}$ then $\operatorname{dim} \mathscr{W}_{\beta}^{\infty}(\Pi(V))=1$.

$A(G(k) \backslash G(\boldsymbol{A}))$ denotes the space of automorphic forms on $G(k) \backslash G(\boldsymbol{A})$. For $f \in A(G(k) \backslash G(A))$, the $\beta$-th Fourier coefficient $W_{\beta}(f)$ is defined by

$$
W_{\beta}(f)(g)=\int_{U(k) \backslash U(\boldsymbol{A})} f(u g) \psi_{\beta}\left(u^{-1}\right) d u .
$$

Denote by $\bar{W}_{\beta}$ the linear functional on $A(G(k) \backslash G(A))$ defined by $f \mapsto W_{\beta}(f)(1)$. It satisfies

$$
\bar{W}_{\beta}(r(u) f)=\psi_{\beta}(u) \bar{W}_{\beta}(f) \quad(u \in U(\boldsymbol{A})) .
$$

Let $(V, h)$ be a $(-1)$-hermitian space over $D$. We write $A^{\infty}(G(k) \backslash G(A))$ for the space of smooth automorphic forms without the $\boldsymbol{K}$-finiteness condition. Let $r$ again denote the right regular action on $A(G(k) \backslash G(A))$ or on $A^{\infty}(G(k) \backslash G(\boldsymbol{A}))$.

By a parallel argument to the first part of the proof of Theorem 2.2 in [Kudla et al. 1992], we have that if $A, B: \Pi(V) \rightarrow A(G(k) \backslash G(A))$ are two intertwining operators then $A$ and $B$ both extend to continuous $G(A)$-intertwining operators $A^{\infty}, B^{\infty}: \Pi(V)^{\infty} \rightarrow A^{\infty}(G(k) \backslash G(\boldsymbol{A}))$. Since $\bar{W}_{\beta}$ can extend to a continuous linear functional on $A^{\infty}(G(k) \backslash G(\boldsymbol{A}))$, we conclude that both $A_{\beta}=\bar{W}_{\beta} \circ A$ and $B_{\beta}=\bar{W}_{\beta} \circ B$ lie in $\mathscr{W}_{\beta}^{\infty}(\Pi(V))$. As in the observation just before Lemma 2.5 in [Kudla et al. 1992], we have the following results. If $\beta \notin \mathcal{O}_{V}$ and $\beta \neq 0$, then $A_{\beta}=B_{\beta}=0$ by Proposition 4.10(i). If $\beta \in \mathbb{O}_{V}$, there is a $c_{\beta} \in \mathbb{C}$ such that $A_{\beta}=c_{\beta} B_{\beta}$ by Proposition 4.10(ii). Moreover, if both $\beta_{1}$ and $\beta_{2}$ lie in the same orbit in $\mathrm{O}_{V}$ then $c_{\beta_{1}}=c_{\beta_{2}}$.

Proposition 4.11. Suppose that $\left\{\left(V_{v}, h_{v}\right)\right\}$ is as in the assumption of Proposition 4.10. Then $\operatorname{dim} \operatorname{Hom}(\Pi(V), A(G(k) \backslash G(\boldsymbol{A}))) \leq 2^{s_{D}-2}$. 
Proof. From the definition of $\mathrm{O}_{V}$ and Proposition 4.3(i), $\mathrm{O}_{V}$ has $2^{s_{D}-2}$ orbits. By the preceding observation and the next lemma, the proposition is proved.

Lemma 4.12. (It is not necessary to impose the assumption of Proposition 4.10.) If $E: \Pi \rightarrow A(G(k) \backslash G(A))$ is an intertwining operator satisfying $\bar{W}_{\beta} \circ E=0$ for all $\beta \in D_{-} \backslash\{0\}$ then $E=0$.

This is shown by the same argument as [Kudla et al. 1992, Lemma 2.5].

Next consider the equality of the relation in Proposition 4.11. For $\phi \in \mathscr{Y}\left(V_{A}\right)$ and $g \in G(A)$, we let

$$
I_{\phi}(g)=\int_{G\left(V_{k}\right) \backslash G\left(V_{A}\right)} \theta(g, l ; \phi) d l,
$$

with the usual theta kernel

$$
\theta(g, l ; \phi)=\sum_{x \in V(k)} \omega_{V}(g) \phi\left(l^{-1} x\right) .
$$

Since $G(V)$ is anisotropic, $I_{\phi}(g)$ is well-defined and $I_{\phi} \in A(G(k) \backslash G(A))$. This defines an intertwining operator $I_{V}$ from $\mathscr{S}\left(V_{A}\right)$ to $A(G(k) \backslash G(A))$. It is $G\left(V_{v}\right)$ invariant for all nonarchimedean places and $\left(\mathfrak{h}_{v}, L_{v}\right)$-invariant for archimedean places. Therefore the image $\operatorname{Im} I_{V}$ of the intertwining operator is isomorphic to a quotient of $\Pi(V)$. Since $\Pi(V)$ is irreducible, $\operatorname{Im} I_{V}$ is isomorphic to $\Pi(V)$. Applying the square integrability criterion, and by Proposition 6.9 in [Kudla et al. 1992], we have that $\operatorname{Im} I_{V} \subset L^{2}(G(k) \backslash G(A))$. For $\beta \in D_{-} \backslash\{0\}$,

$$
\begin{aligned}
W_{\beta}\left(I_{\phi}\right)(g) & =\int_{U(k) \backslash U(\boldsymbol{A})} I_{\phi}(u g) \psi_{\beta}\left(u^{-1}\right) d u \\
& =\int_{U(k) \backslash U(\boldsymbol{A})} d u \int_{G\left(V_{k}\right) \backslash G\left(V_{A}\right)} \sum_{x \in V(k)} \omega_{V}(u g) \phi\left(l^{-1} x\right) \psi_{\beta}\left(u^{-1}\right) d l \\
& =\int_{G\left(V_{k}\right) \backslash G\left(V_{A}\right)} \sum_{h(x, x)=\beta} \omega_{V}(g) \phi\left(l^{-1} x\right) d l .
\end{aligned}
$$

In particular, if $\beta$ is not represented by $h$ then $W_{\beta}\left(I_{\phi}\right)=0$. If $\beta=h\left(x_{0}, x_{0}\right)$ for some $x_{0} \in V \backslash\{0\}$ then

$$
W_{\beta}\left(I_{\phi}\right)(g)=\int_{G\left(V_{A}\right)} \omega_{V}(g) \phi\left(l^{-1} x_{0}\right) d l .
$$

Choosing a suitable $\phi$ we have $W_{\beta}\left(I_{\phi}\right) \neq 0$. In particular, if $V_{1}, \ldots, V_{n}$ are not isometric to each other then $I_{V_{1}}, \ldots, I_{V_{n}}$ are linearly independent. Thus we have obtained the following theorem.

Theorem 4.13. Suppose that $\left\{\left(V_{v}, h_{v}\right)\right\}$ is as in the assumption of Proposition 4.10. Then $\operatorname{dim} \operatorname{Hom}(\Pi(V), A(G(k) \backslash G(\boldsymbol{A})))=2^{s_{D}-2}$. 
Proposition 4.14. Suppose that there are no global $(-1)$-hermitian space $(V, h)$ such that $\left\{\left(V_{v}, h_{v}\right)\right\}_{v}$ is given by the completions of $(V, h)$. Then

$$
\operatorname{Hom}(\Pi, A(G(k) \backslash G(\boldsymbol{A})))=0 .
$$

Proof. Let $E: \Pi \rightarrow A(G(k) \backslash G(\boldsymbol{A}))$ be a nonzero intertwining operator. There exists $\beta \in D_{-} \backslash\{0\}$ such that $E_{\beta}=\bar{W}_{\beta} \circ E$ is nonzero by Lemma 4.12. Therefore for any $v$, the restriction of $E_{\beta}$ to $R\left(V_{v}\right)$ is nonzero. By Lemma 4.8 and $4.9\langle\beta\rangle \simeq V_{v}$. This contradicts the assumption for $\left\{\left(V_{v}, h_{v}\right)\right\}$.

Let $\left\{\left(V_{v}, h_{v}\right)\right\}$ be given by the completions at all $v$ for some $(-1)$-hermitian right $D$-module $(V, h)$ with rank one and det $V \neq 0$. We define the quadratic character $\chi_{V}$ of $\boldsymbol{A}^{\times} / k^{\times}$by $\chi_{V}=\prod_{v} \chi_{V_{v}}$. Since

$$
-v\left(D_{-} \backslash\{0\}\right)=\left\{\alpha \in k^{\times} \mid \alpha \notin\left(k_{v}^{\times}\right)^{2} \text { for all } v \in S_{D}\right\},
$$

any $\omega_{k^{\prime} / k}$ appearing in Proposition 3.2(ii) can be written by the form $\chi_{V}$ for some $V$. By the definition of $R\left(V_{v}\right)$ we have

$$
\bigotimes_{v} R\left(V_{v}\right) \subset \operatorname{Ind}_{P(\boldsymbol{A})}^{G(\boldsymbol{A})}\left(\left(\chi_{V}|\cdot|^{-1 / 2}\right) \circ v\right) .
$$

On the other hand, by Proposition 1.1, 1.2 in [Kudla et al. 1992] and Proposition 4.5, 4.7, any irreducible constituent of $\operatorname{Ind}_{P(\boldsymbol{A})}^{G(\boldsymbol{A})}\left(\left(\chi_{V}|\cdot|^{-1 / 2}\right) \circ v\right)$ is written by the form $\bigotimes_{v} R\left(\widetilde{V}_{v}\right)$ where $\left\{\widetilde{V}_{v}\right\}_{v}$ is a collection of $(-1)$-hermitian right $D_{v^{-}}$ modules with rank one and $\operatorname{det} \widetilde{V}_{v}=\operatorname{det} V_{v}$ for all $v$. Therefore Proposition 4.14 and Theorem 4.13 conclude that the representations obtained by the images of $N\left(w_{0},\left(\chi_{V}|\cdot|_{\boldsymbol{A}}^{1 / 2}\right) \circ v\right)$ are exhausted by the representations given by Theorem 4.1(3).

\section{Arthur parameters for residual spectrum}

Here we give an expectation about the Arthur parameters for the residual spectrum of $G$. The dual group $\widehat{G}$ of $G$ equals $S O(5, \mathbb{C})$, which we realize as

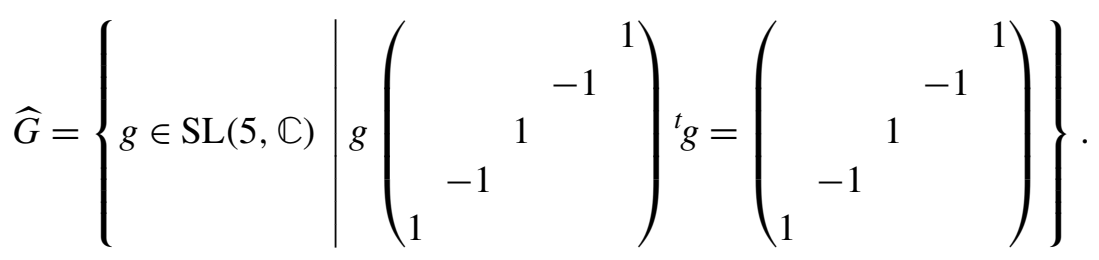

For each $v$ we denote by $W_{k_{v}}$ the Weil group of $k_{v}$ and write ${ }^{L} G_{v}$ for the $L$-group $\widehat{G} \times W_{k_{v}}$ of $G_{v}=G \otimes_{k} k_{v}$. We write $\mathscr{L}_{v}$ for the Langlands group introduced in 
[Kottwitz 1984]:

$$
\mathscr{L}_{v}:= \begin{cases}W_{k_{v}} & \text { if } k_{v} \text { is archimedean, } \\ W_{k_{v}} \times S U(2, \mathbb{R}) & \text { if } k_{v} \text { is nonarchimedean }\end{cases}
$$

A local Arthur parameter for $G$ is a continuous homomorphism

$$
\psi: \mathscr{L}_{v} \times \mathrm{SL}(2, \mathbb{C}) \rightarrow{ }^{L} G_{v}
$$

such that (i) $\left.\psi\right|_{W_{k_{v}}}$ is semisimple and has bounded image, (ii) the composition

$$
W_{k_{v}} \stackrel{\psi}{\rightarrow}{ }^{L} G_{v} \stackrel{\mathrm{pr}_{2}}{\rightarrow} W_{k_{v}}
$$

is the identity, and (iii) $\psi$ restricted to $\operatorname{SL}(2, \mathbb{C})$ or $S U(2) \times \operatorname{SL}(2, \mathbb{C})$ is analytic.

Expectation 5.1. Let $\pi=\bigotimes_{v} \pi_{v}$ be a residual discrete representation appearing in Theorem 4.1. The Arthur parameter $\psi_{\pi_{v}}$ associated to $\pi_{v}$ is given by the following.

(1) For $\mathbf{1}_{G(\boldsymbol{A})}$ we have $\psi_{\mathbf{1}_{G(\boldsymbol{A})}} \mid \mathscr{L}_{v}=1_{5} \times \mathrm{pr}_{W_{k_{v}}}$ and

$$
\psi_{\mathbf{1}_{G(A)}}\left(\left(\begin{array}{cc}
a & x \\
y & -a
\end{array}\right)\right)=\left(\begin{array}{ccccc}
4 a & x & & & \\
2 y & 2 a & x & & \\
& 2 y & 0 & x & \\
& & 2 y & -2 a & x \\
& & & 2 y & -4 a
\end{array}\right) \in \operatorname{Lie} \widehat{G} .
$$

(2) For $J_{P}^{G}(\pi)$ we have

$$
\psi_{J_{P}^{G}(\pi)} \mid \mathscr{L}_{v}=\left(\begin{array}{l|l|l}
\varphi_{\pi_{v}} & & \\
\hline & 1 & \\
\hline & & \operatorname{Ad}(J)^{t} \varphi_{\pi_{v}}^{-1}
\end{array}\right) \times \operatorname{pr}_{W_{k_{v}}}
$$

and

$$
\psi_{J_{P}^{G}(\pi)}\left(\left(\begin{array}{cc}
a & x \\
y & -a
\end{array}\right)\right)=\left(\begin{array}{cc|c|cc}
a & & x & \\
& a & & & x \\
\hline & 0 & & \\
\hline y & & -a & \\
& y & & -a
\end{array}\right) \in \operatorname{Lie} \widehat{G} .
$$

Here $\varphi_{\pi_{v}}$ is the Langlands parameter for $\pi_{v}$. Since $\pi_{v}$ is self-dual the image of $\varphi_{\pi_{v}}$ is contained in $\operatorname{SL}(2, \mathbb{C}) \times W_{k_{v}}$.

(3) For $R(V)$ we have

$$
\psi_{R\left(V_{v}\right) \mid \mathscr{L}_{v}}=\left(\begin{array}{ccccc}
\chi_{V_{v}} & & & & \\
& c_{1} & & c_{2} & \\
& & \chi_{V_{v}} & & \\
& c_{3} & & c_{4} & \\
& & & & \chi_{V_{v}}
\end{array}\right) \times \operatorname{pr}_{W_{k_{v}}}
$$


and

$$
\psi_{R\left(V_{v}\right)}\left(\left(\begin{array}{cc}
a & x \\
y & -a
\end{array}\right)\right)=\left(\begin{array}{cc|c|cc}
2 a & 0 & x & & \\
0 & 0 & 0 & & \\
\hline 2 y & 0 & 0 & 0 & -x \\
\hline & 0 & 0 & 0 \\
& & -2 y & 0 & -2 a
\end{array}\right) \in \operatorname{Lie} \widehat{G},
$$

where

$$
\left(\begin{array}{ll}
c_{1}(w) & c_{2}(w) \\
c_{3}(w) & c_{4}(w)
\end{array}\right)=\left\{\begin{array}{cl}
1_{2} & \text { if } w \in W_{k_{v}^{\prime}}, \\
\left(\begin{array}{ll}
0 & 1 \\
1 & 0
\end{array}\right) & \text { if } w \in W_{k_{v}} \backslash W_{k_{v}^{\prime}}
\end{array}\right.
$$

for the quadratic extension $k^{\prime}$ of $k$ attached to $\chi_{V}$.

Remark 5.2. The (global) Arthur parameter of $\mathbf{1}_{G(\boldsymbol{A})}$ should coincide with that of $\mathbf{1}_{\mathrm{Sp}(2, \boldsymbol{A})}$. For $J_{P}^{G}(\pi)$ the unique irreducible quotient $J_{P_{1}}^{\mathrm{Sp}(2)}(J L(\pi))$ of

$$
\operatorname{Ind}_{P_{1}(\boldsymbol{A})}^{\mathrm{Sp}(2, \boldsymbol{A})}\left(J L(\pi)|\operatorname{det}|_{\boldsymbol{A}}^{1 / 2}\right)
$$

occurs in the residual spectrum of $\operatorname{Sp}(2)$ where $P_{1}$ is the Siegel parabolic subgroup of $\mathrm{Sp}(2)$ and $J L(\pi)$ is the Jacquet-Langlands correspondence of $\pi$ [Kim 1995]. The Arthur parameters of $J_{P}^{G}(\pi)$ and $J_{P_{1}}^{\mathrm{Sp}(2)}(J L(\pi))$ should coincide. For $R(V)$ the theta lift of the trivial representation of the orthogonal group of a 2-dimensional quadratic space with determinant det $V$ occurs in the residual spectrum of $\operatorname{Sp}(2)$ [Kon-no 1994] and its Arthur parameter should coincide with that of $R(V)$.

\section{Acknowledgment}

The author thanks Professor Takuya Kon-no for much helpful advice and encouragement.

\section{References}

[Godement and Jacquet 1972] R. Godement and H. Jacquet, Zeta functions of simple algebras, Lecture Notes in Mathematics 260, Springer, Berlin, 1972. MR 49 \#7241 Zbl 0244.12011

[Harish-Chandra 1968] Harish-Chandra, Automorphic forms on semisimple Lie groups, Lecture Notes in Mathematics 62, Springer, Berlin, 1968. MR 38 \#1216 Zbl 0186.04702

[Jacquet and Langlands 1970] H. Jacquet and R. P. Langlands, Automorphic forms on GL(2), Lecture Notes in Mathematics 114, Springer, Berlin, 1970. MR 53 \#5481 Zbl 0236.12010

[Johnson 1990] K. D. Johnson, "Degenerate principal series and compact groups", Math. Ann. 287:4 (1990), 703-718. MR 91h:22028 Zbl 0687.22003

[Kim 1995] H. H. Kim, “The residual spectrum of $\mathrm{Sp}_{4}$ ”, Compositio Math. 99:2 (1995), 129-151. MR 97c:11056 Zbl 0877.11030

[Knapp and Stein 1980] A. W. Knapp and E. M. Stein, "Intertwining operators for semisimple groups, II”, Invent. Math. 60:1 (1980), 9-84. MR 82a:22018 Zbl 0454.22010 
[Knus 1991] M.-A. Knus, Quadratic and Hermitian forms over rings, Grundlehren der Math. Wissenschaften 294, Springer, Berlin, 1991. MR 92i:11039 Zbl 0756.11008

[Kon-no 1994] T. Kon-no, “The residual spectrum of Sp(2)", Proc. Japan Acad. Ser. A Math. Sci. 70:6 (1994), 204-207. MR 96a:11048 Zbl 0833.11018

[Kottwitz 1984] R. E. Kottwitz, "Stable trace formula: cuspidal tempered terms", Duke Math. J. 51:3 (1984), 611-650. MR 85m:11080 Zbl 0576.22020

[Kudla 1994] S. S. Kudla, "Splitting metaplectic covers of dual reductive pairs", Israel J. Math. 87:1-3 (1994), 361-401. MR 95h:22019 Zbl 0840.22029

[Kudla et al. 1992] S. S. Kudla, S. Rallis, and D. Soudry, "On the degree 5 L-function for $\operatorname{Sp}(2)$ ", Invent. Math. 107:3 (1992), 483-541. MR 93b:11061 Zbl 0776.11028

[Langlands 1971] R. P. Langlands, Euler products, Yale Mathematical Monographs 1, Yale University Press, New Haven, Conn., 1971. MR 54 \#7387 Zbl 0231.20016

[Mœglin and Waldspurger 1995] C. Mœglin and J.-L. Waldspurger, Spectral decomposition and Eisenstein series, Cambridge Tracts in Mathematics 113, Cambridge Univ. Press, Cambridge, 1995. MR 97d:11083 Zbl 0846.11032

[Mœglin et al. 1987] C. Mœglin, M.-F. Vignéras, and J.-L. Waldspurger, Correspondances de Howe sur un corps p-adique, Lecture Notes in Mathematics 1291, Springer, Berlin, 1987. MR 91f:11040 Zbl 0642.22002

[Muić and Savin 2000] G. Muić and G. Savin, "Complementary series for Hermitian quaternionic groups”, Canad. Math. Bull. 43:1 (2000), 90-99. MR 2001g:22019 Zbl 0945.22005

[Rallis 1987] S. Rallis, L-functions and the oscillator representation, Lecture Notes in Mathematics 1245, Springer, Berlin, 1987. MR 89b:11046 Zbl 0605.10016

[Scharlau 1985] W. Scharlau, Quadratic and hermitian forms, Grundlehren der Mathematischen Wissenschaften 270, Springer, Berlin, 1985. MR 86k:11022 Zbl 0584.10010

[Shahidi 1981] F. Shahidi, "On certain L-functions", Amer. J. Math. 103:2 (1981), 297-355. MR 82i:10030 Zbl 0467.12013

[Shahidi 1990] F. Shahidi, "A proof of Langlands' conjecture on Plancherel measures; complementary series for p-adic groups", Ann. of Math. (2) 132:2 (1990), 273-330. MR 91m:11095 Zbl 0780.22005

[Silberger 1979] A. J. Silberger, Introduction to harmonic analysis on reductive p-adic groups, Math. Notes 23, Princeton Univ. Press, Princeton, N.J., 1979. MR 81m:22025 Zbl 0458.22006

[Zhu 1992] C.-b. Zhu, "Invariant distributions of classical groups", Duke Math. J. 65:1 (1992), 85119. MR 92k:22022 Zbl 0764.22009

Received March 19, 2006. Revised August 28, 2006.

TAKANORI YASUDA

GRADUATE SCHOOL OF MATHEMATICS

KYUSHU UNIVERSITY 33

FUKUOKA 812-8581

JAPAN

tyasuda@math.kyushu-u.ac.jp 